20

\title{
A Multi-Sampling Ionization Chamber (MUSIC) for Measurements of Fusion Reactions with Radioactive Beams
}

\author{
P.F.F. Carnelli ${ }^{\mathrm{a}, \mathrm{b}, \mathrm{c}}$, S. Almaraz-Calderon ${ }^{\mathrm{a}, 1}$, K.E. Rehm ${ }^{\mathrm{a}, *}$, M. Albers $^{\mathrm{a}, 2}, \mathrm{M}$. \\ Alcorta $^{\mathrm{a}, 3}$, P.F. Bertone ${ }^{\mathrm{a}, 4}$, B. Digiovine ${ }^{\mathrm{a}}$, H. Esbensen ${ }^{\mathrm{a}}$, J. Fernández Niello ${ }^{\mathrm{b}, \mathrm{d}}$, D. \\ Henderson $^{\mathrm{a}}$, C.L. Jiang ${ }^{\mathrm{a}}$, J. Lai ${ }^{\mathrm{e}}$, S.T. Marley ${ }^{\mathrm{a}, 5}$, O. Nusair ${ }^{\mathrm{a}}$, T. Palchan-Hazan ${ }^{\mathrm{a}}$, \\ R.C. Pardo ${ }^{\mathrm{a}}$, M. Paul ${ }^{\mathrm{f}}$, and C. Ugalde \\ ${ }^{a}$ Physics Division, Argonne National Laboratory, Argonne, Illinois 60439, USA \\ ${ }^{\mathrm{b}}$ Laboratorio TANDAR, Comisión Nacional de Energía Atómica, Av. Gral. Paz 1499, \\ B1650KNA, San Martín, Buenos Aires, Argentina \\ ${ }^{\mathrm{c} C o n s e j o}$ Nacional de Investigaciones Científicas y Técnicas, Av. Rivadavia 1917, C1033AAJ, \\ Buenos Aires, Argentina \\ ${ }^{\mathrm{d}}$ Universidad Nacional de San Martín, Campus Miguelete, B1650BWA, San Martín, Buenos \\ Aires, Argentina \\ ${ }^{\mathrm{e}}$ Department of Physics and Astronomy, Louisiana State University, Baton Rouge, Louisiana \\ 70803, USA \\ ${ }^{\mathrm{f}}$ Racah Institute of Physics, Hebrew University, Jerusalem, Israel
}

*Corresponding author Tel.: +1 (630) 252-4073, Fax: +1 (630) 252-6210, e-mail: $\underline{\text { rehm@anl.gov }}$

PACS: 25.60.Pj, 29.40.Cs; 26.30.Ca; 29.38.Gj

Keywords: Active Target Detector; Multi-Sampling Ionization Chamber; Fusion Reactions

Abstract

\footnotetext{
${ }^{1}$ Present address: Department of Physics, Florida State University, Tallahassee, Florida 32306, USA.

${ }^{2}$ Present address: Ernst \& Young GmbH, 65760 Eschborn, Germany.

${ }^{3}$ Present address: TRIUMF, Vancouver, British Columbia VGT 2A3, Canada.

${ }^{4}$ Present address: Marshall Space Flight Center, Huntsville, Alabama 35812, USA.

${ }^{5}$ Present address: Department of Physics, University of Notre Dame, Notre Dame, Indiana 46556, USA.
} 
A detection technique for high-efficiency measurements of fusion reactions with low-intensity radioactive beams was developed. The technique is based on a Multi-Sampling Ionization Chamber (MUSIC) operating as an active target and detection system, where the ionization gas acts as both target and counting gas. In this way, we can sample an excitation function in an energy range determined by the gas pressure, without changing the beam energy. The detector provides internal normalization to the incident beam and drastically reduces the measuring time. In a first experiment we tested the performance of the technique by measuring the ${ }^{10,13,15} \mathrm{C}+{ }^{12} \mathrm{C}$ fusion reactions at energies around the Coulomb barrier.

\section{Introduction}

Fusion reactions play an important role in nature, starting from the creation of the light elements in the Big Bang. They are crucial for the production of heavier elements in the stars' quiescent burning phase as well as in stellar explosions. Fusion processes also generate the energy in the Sun that created and maintains life as we know it. In nuclear physics, fusion reactions can produce exotic nuclei away from stability on the proton-rich side of the mass valley and they are crucial for the production of very heavy nuclei. The availability of radioactive beams at first-generation facilities over the last 20 years has opened many new possibilities for the study of fusion reactions. Particularly, for nuclear structure studies reactions with unstable nuclei offer the possibility to reach even further away from the valley of stability.

Fusion between carbon isotopes has attracted the attention of physicists and astronomers for the last 50 years. From fundamental nuclear structure effects to recent discoveries in stellar phenomena, there are still many open questions. One of them is the presence of oscillations in the ${ }^{12} \mathrm{C}+{ }^{12} \mathrm{C}$ fusion cross sections that are not observed in neighboring systems and are not completely understood yet [1]. In nuclear astrophysics, fusion reactions involving neutron-rich carbon isotopes may play a fundamental role opening new paths for nucleosynthesis in x-ray binaries, where new explosive phenomena called superbursts have recently been observed [2].

In most cases, fusion reactions in the laboratory are studied via the identification of the evaporation residues (ERs) using a variety of experimental techniques. The identification of the ERs can be achieved by detecting the decay radiation from characteristic energy levels or by direct particle detection. For the first case, $\gamma$ - (e.g., Refs. [3-5]), $\beta$ - (e.g., Ref. [6]), or x-ray measurements (e.g., Refs. [7-9]) are frequently employed. For the second case, the ERs can be directly detected in $\Delta E-E_{\text {res }}$ detector telescopes mounted at small scattering angles. For that, thin Si $\Delta E$ detectors or ionization chambers have been used (see, for example, Refs. $[1,10])$. Time-of-flight (ToF) techniques, i.e. measuring the velocities and the energy of the ERs, or gas-filled separators [11] have also been employed in the past.

By using one of these methods, excitation functions are obtained by varying the energy of the beam and using large high-efficiency detector arrays (in the decay radiation case), or measuring angular distributions of the ERs (in direct measurements). These experiments require a good relative normalization between the measurements made at different energies. For experiments with low-intensity radioactive beams the measurements can be very time consuming since typically only one cross section is obtained at each bombarding energy. 
As an alternative, a Multi-Sampling Ionization Chamber (MUSIC) is presented here. The MUSIC is a small gas-filled detector which operates at low beam intensities $\left(\sim 10^{4}\right.$ particles/s), counting both incident particles and reaction products. It is also capable of measuring several energy points of an excitation function at once. The detector operates as an active target, i.e. the ionization gas serves as target for the reaction and as detection medium at the same time. The projectile loses energy along its path inside the detector and fusion occurs with different energies depending on the position inside the detector. Since an ER is heavier than a beam particle, it will suffer a significantly larger energy loss. Therefore, the detected energy loss $\Delta E$ will suddenly increase at the position where a fusion reaction occurred and the ER will stop inside the detector, which is the signature of a fusion event.

Multi-sampling detectors have been employed in the past for the study of heavy-ion reactions. A multisampling ionization chamber was first developed for measurements with relativistic heavy ions [12]. Later multi-sampling proportional counters $[13,14]$ were built for experiments with relativistic as well as with low-energy heavy ions. All these detectors, however, are generally quite large (typically $1 \mathrm{~m}$ long) which can lead to complications when isotopically enriched gases are to be used as an active target.

This paper is organized as follows: in Sect. 2 we give details of the main design features and the operational principle of the MUSIC detector. In Sect. 3 we describe the experimental setup for using the detector at the ATLAS facility at Argonne National Laboratory and the general procedure for its operation, whereas the performance of the technique under real experimental conditions is given in Sect. 4. A summary and future perspectives are presented in Sect. 5.

\section{Schematic and operational principle}

The schematic of the multi-sampling ionization chamber MUSIC is shown in Fig. 1. The ionization chamber is mounted inside a $30 \mathrm{~cm}(\mathrm{~L}) \times 10 \mathrm{~cm}(\mathrm{~W}) \times 20 \mathrm{~cm}(\mathrm{H})$ aluminum box that can be filled with a suitable counting gas $\left(\mathrm{He}, \mathrm{CH}_{4}, \mathrm{Ne}, \mathrm{Ar}\right)$. In the following we discuss as an example the fusion reactions between ${ }^{10,13,15} \mathrm{C}$ and ${ }^{12} \mathrm{C}$ studied by detecting the evaporation residues in MUSIC, which was filled with $\mathrm{CH}_{4}$ at 200 mbar thus providing the ${ }^{12} \mathrm{C}$ target. The beams, ${ }^{10,13,15} \mathrm{C}$, were obtained from the heavy ion accelerator ATLAS. Beams of the stable ${ }^{13} \mathrm{C}$ were accelerated in a tandem Van de Graaff accelerator, while beams of radioactive ${ }^{10,15} \mathrm{C}$, with half-lives of $19.3 \mathrm{~s}$ and $2.46 \mathrm{~s}$, respectively, were obtained via the in-flight method [15]. The beam production technique is discussed in Sect. 3.3 and more details can be found in Ref. [15]. The ${ }^{10,13,15} \mathrm{C}$ beams enter and exit the detector through $1.45 \mathrm{mg} / \mathrm{cm}^{2}$ thick titanium windows. Titanium was chosen to minimize the production of ERs in the window material. In this experiment we have used non-enriched methane $\left(\mathrm{CH}_{4}\right)$ gas as active target, for its good counting properties and because reactions with the hydrogen do not interfere with the events of interest at the energies studied in this experiment. The correction for the $1 \%$ contamination from ${ }^{13} \mathrm{C}$ was in all cases smaller than the statistical uncertainty of the measured fusion cross sections.

In order to obtain the multi-sampling capabilities of the MUSIC detector, the anode of the ionization chamber is segmented in a pattern shown in the lower part of Fig. 1. There are a total of 18 strips numbered from 0 to 17, with each strip being $1.58 \mathrm{~cm}$ long. The "active strips" are numbered from 1 to 16 , while the first and last strips, $S_{0}$ and $S_{17}$, serve as so-called "control strips". While the segmentation 
chosen for this detector does not allow a full tracking of the individual particles, it allows a clean identification of fusion events as well as the elimination of elastic and inelastic scattering events based on their multiplicity. This will be discussed below.

The energy signals from the anode were read out using Mesytec MPR-16 preamplifiers and Mesytec MSCF-16 shaper amplifiers [16]. In addition there were four more energy signals from the control strips $\mathrm{S}_{0}$ and $\mathrm{S}_{17}$, from the Frisch grid and from the cathode of the ionization chamber that were read out with standard NIM electronics.

\section{MUSIC}

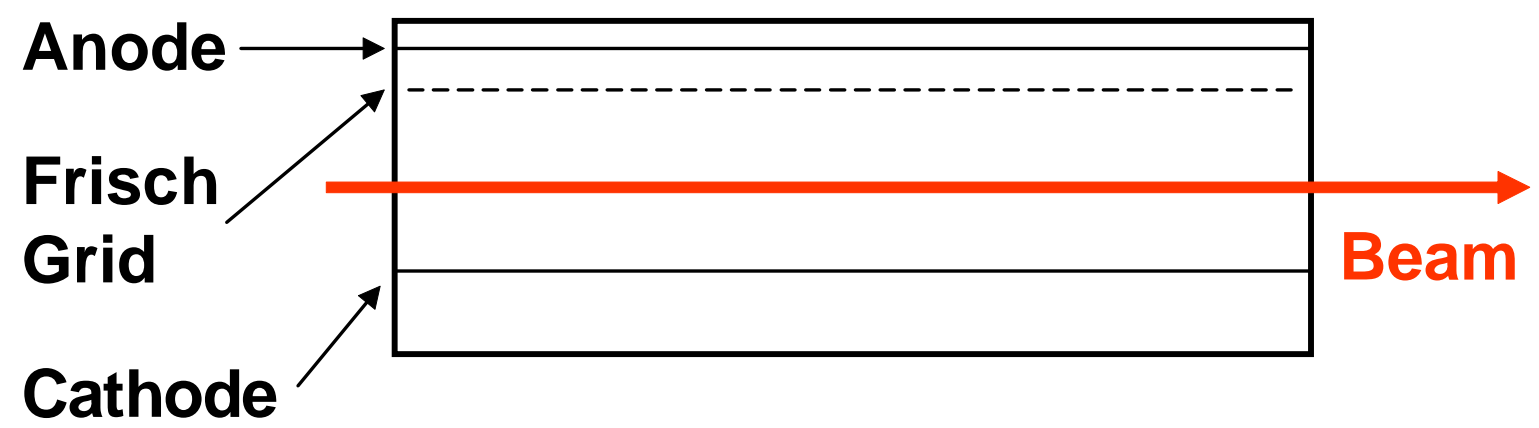

\section{Schematic of the anode structure}

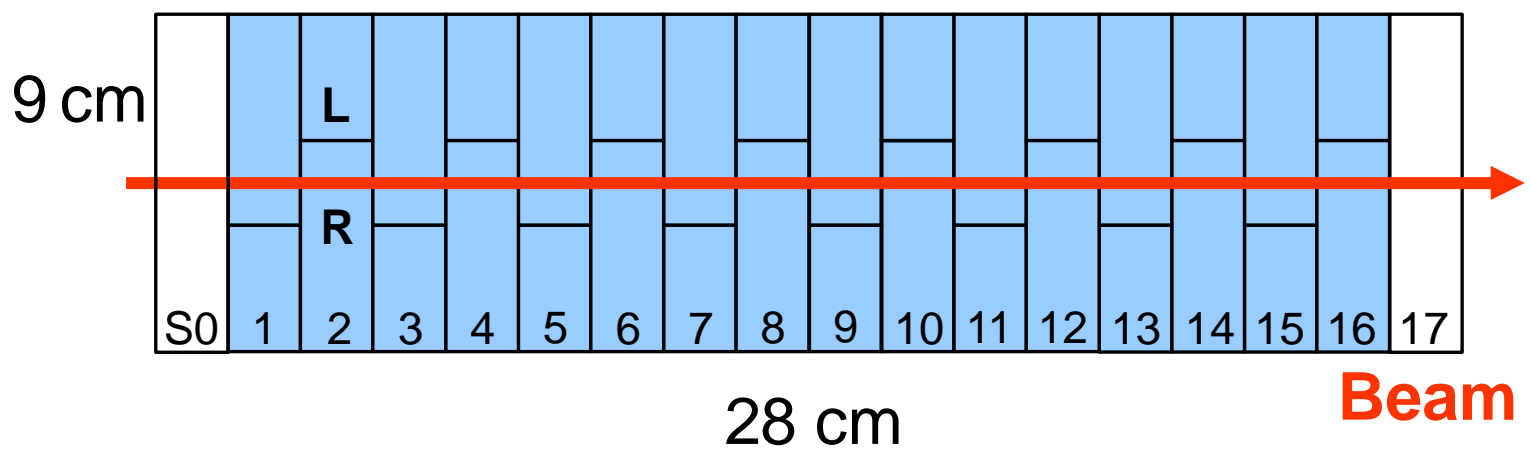

Fig. 1 (color online). Schematic of the Multi-Sampling Ionization Chamber (MUSIC). In the upper panel there is a lateral view of the detector and in the lower panel the structure of the anode.

The information provided by the MUSIC detector for a single incoming particle is best represented in a plot of $\Delta E$ vs. strip number, which is called a "trace". As an example, Fig. 2 shows some experimental traces measured for the ${ }^{13} \mathrm{C}+{ }^{12} \mathrm{C}$ system with a ${ }^{13} \mathrm{C}$ beam at $E_{\text {lab }}=45 \mathrm{MeV}$. The beam reaches the first active strip $\left(S_{1}\right)$ with an energy of $39 \mathrm{MeV}$ and loses a total of $19 \mathrm{MeV}$ in the active volume of gas providing the signals $S_{1}-S_{16}$. Thus, at this beam energy and gas pressure (200 mbar), the excitation 
function of ${ }^{13} \mathrm{C}+{ }^{12} \mathrm{C}$ fusion is being sampled in the c.m. energy range of $10-18 \mathrm{MeV}$ in 16 steps of about $0.5 \mathrm{MeV}$ each. If it does not undergo a nuclear reaction, the ${ }^{13} \mathrm{C}$ beam deposits on average about $1.2 \mathrm{MeV}$ in each strip and, hence, a signal of a beam particle passing through the MUSIC consists of 18 consecutive signals of 1.0 to $1.4 \mathrm{MeV}$ each, as can be seen by the black lines in Fig. 2.

A fusion reaction occurring e.g. in strip 3 (see red lines in Fig. 2) starts as beam-like ${ }^{13} \mathrm{C}$ signals followed by a sudden increase in $\Delta E$ (caused by the higher nuclear charge and mass of the ER), and finishes with no signals because the ER is stopped inside the detector.

Since angular distributions of ERs from fusion reactions in this mass and energy range generally peak at $0^{\circ}$ in the laboratory frame and extend to scattering angles of about $35^{\circ}$ (see e.g. Ref. [1]) practically the full yield of the fusion reactions can be detected in the first 10-12 strips. Fusion events occurring in the last 4-6 strips will experience some losses in efficiency.
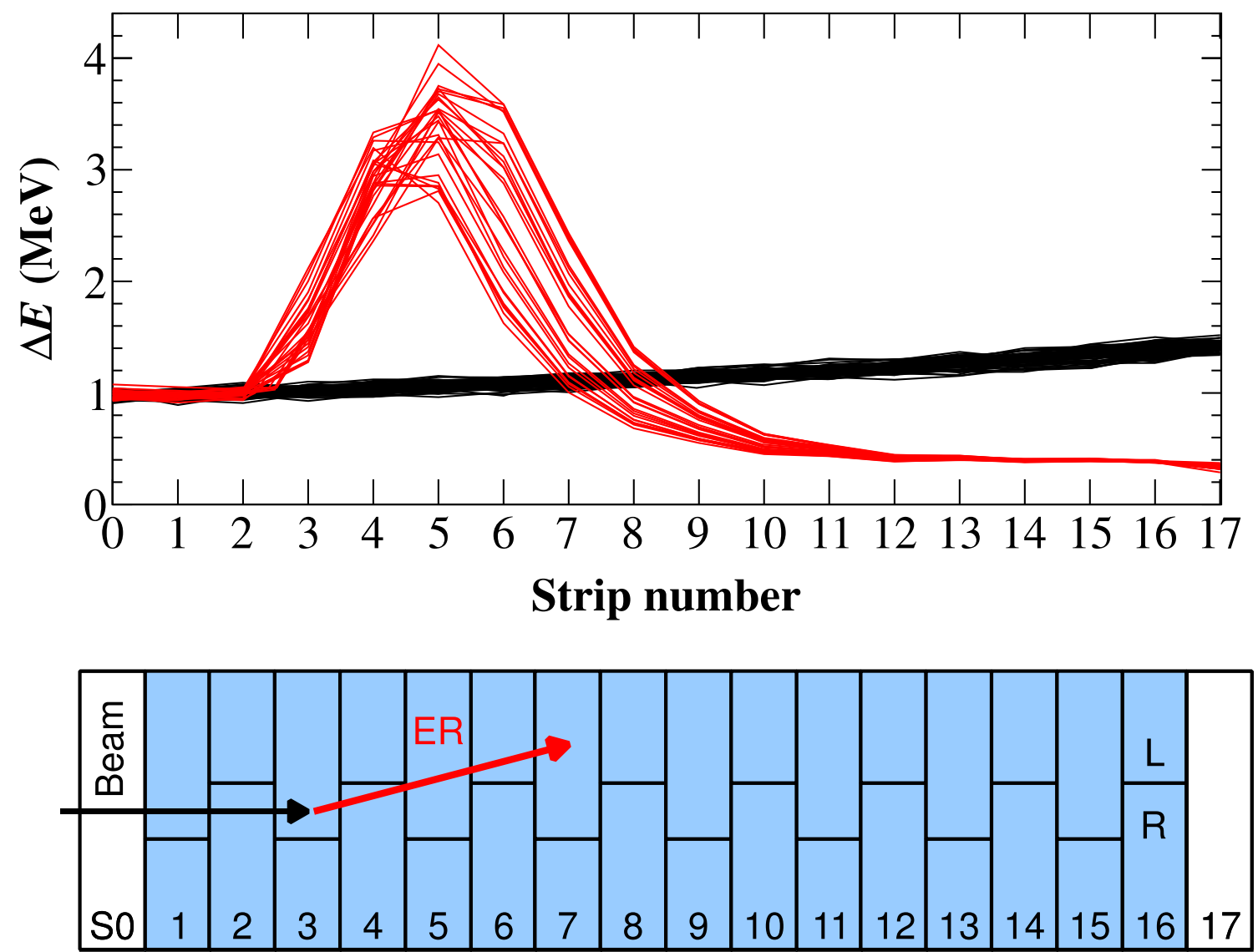

Fig. 2 (color online). Experimental traces measured with the MUSIC detector. Black lines correspond to beam particles interacting with the gas target only by ionization. The red lines correspond to fusion events occurring in 
The signals from anode strips 0 and 17, at the beginning and at the end of the ionization chamber, are used for particle identification (see Sect. 3.3) or as veto signals to eliminate events occurring in the entrance and exit windows.

Another advantage of the multi-sampling method is that the fusion measurement is self-normalizing, since the beam and the fusion events at the various energies are obtained by the same detector using the same number of incident particles without the need to change the energy or using a monitor detector.

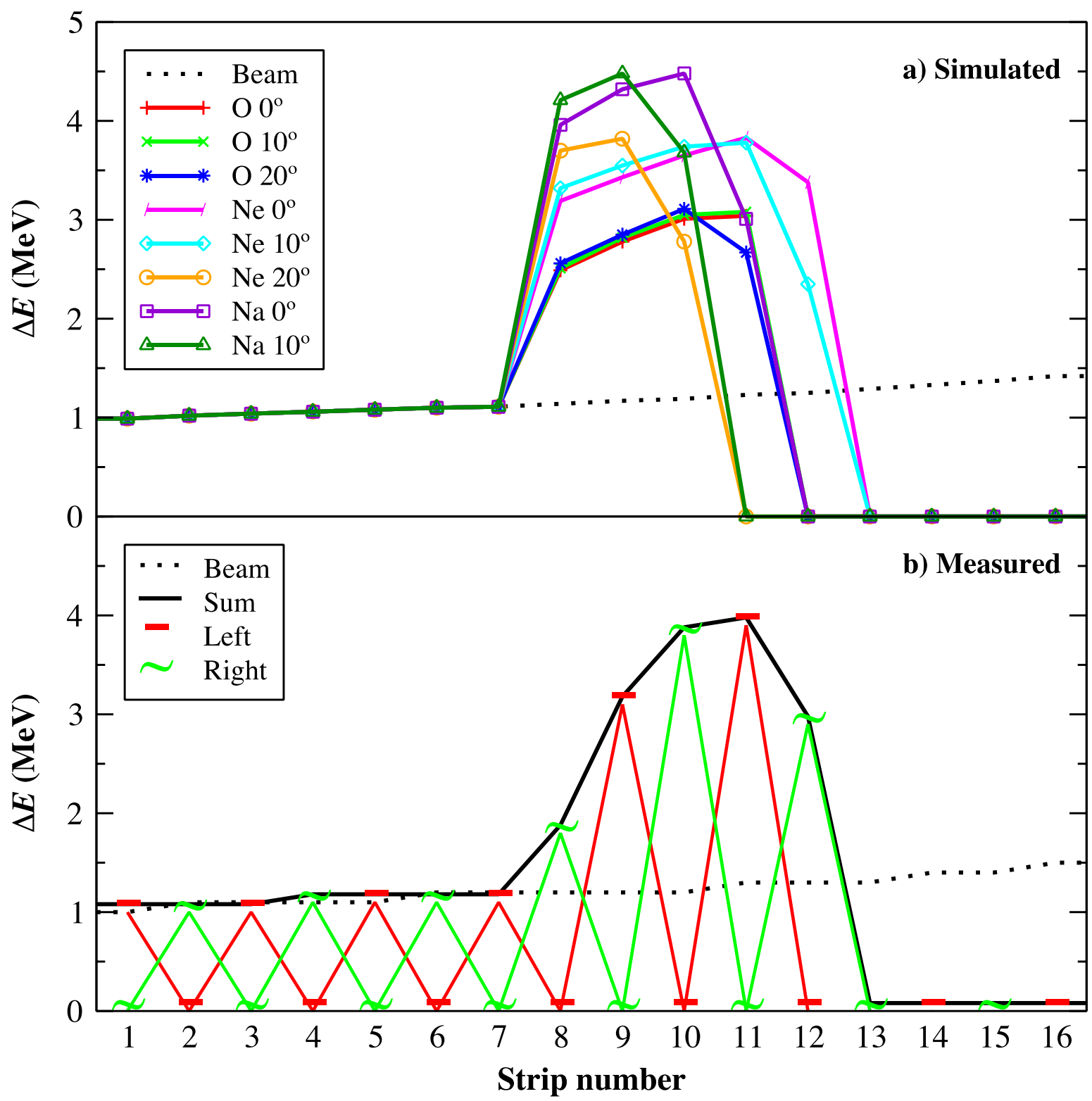

Fig. 3 (color online). Comparison between simulated and experimental fusion signals occurring in strip number 8: a) fusion events simulated with FLUKA, b) left- and right-side signals for a measured fusion event. The "zigzag" structure in the left (red) and right (green) signals is the main feature of a multiplicity-one event. 


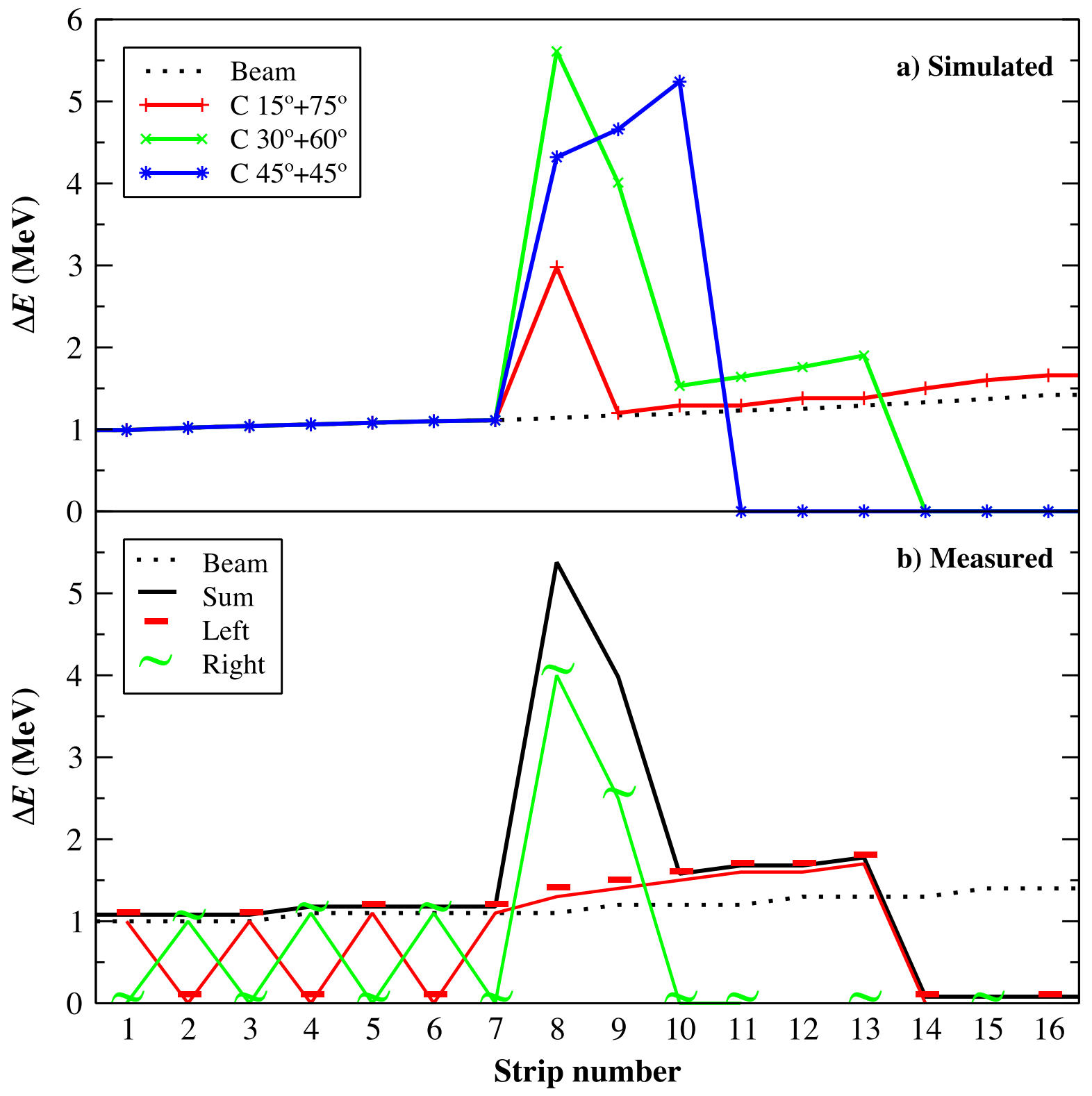

142

Fig. 4 (color online). Simulated and experimental traces for scattering events occurring in strip number 8: a) scattering events simulated with FLUKA, b) a scattering event measured with the MUSIC detector. The simulated signals in a) correspond to two carbon nuclei scattered at angles that add up to approximately $90^{\circ}\left(15^{\circ}+75^{\circ}, 30^{\circ}+60^{\circ}\right.$ and $45^{\circ}+45^{\circ}$ ). In b), note that after the beam-like pattern, there are signals in both sides of the same strip. This is an example of a multiplicity-two event.

In order to eliminate elastic or inelastic scattering events, which might interfere with fusion events, the anode strips 1-16 are subdivided into two staggered sections (marked " $L$ " and " $R$ " in the lower panels of Figs. 1 and 2). The two sections are used as a multiplicity filter. Since light particles (such as p, $\alpha$ ) evaporated by the compound nucleus generate signals that are below the detection threshold, fusion events have multiplicity one (i.e., signals that occur either in the right or in the left side of the anode 
strips). Elastic and inelastic scattering events on the other hand have two particles in the outgoing channel producing events that can be rejected using the "beam-right" and "beam-left" information.

The response of the MUSIC detector to various reaction types was simulated using the Monte Carlo code FLUKA [17]. Results from the simulation of a $45 \mathrm{MeV}{ }^{13} \mathrm{C}$ beam interacting with $\mathrm{CH}_{4}$ are presented in Figs. 3 and 4. Figure 3 shows simulated (a) and experimental (b) traces of fusion (multiplicity-one) events. Fusion events have a pulse height that is typically a factor of 3-4 higher than the pulse height produced by a beam-like particle. Although the FLUKA simulation gives an indication of a grouping of the pulse height according to the nuclear charge of the ERs, variations of the emission angle and of the location of the fusion reaction in a strip smear out this grouping. Simulated traces of events originating from elastic/inelastic scattering at a few scattering angles are shown in Fig. 4a. Since for scattering of same-mass particles the relative angle of the two particles is approximately $90^{\circ}$ in the laboratory system, events from e.g. $\pm 45^{\circ}$ or $+75^{\circ}$ and $-15^{\circ}$ need to be summed as shown in Fig. 4a. These events also show a jump in the trace as observed for fusion, but as shown in Fig. $4 \mathrm{~b}$ they produce signals on both sides of the same strip.

\section{Experimental details}

\subsection{Ionization chamber}

For the experiments described in this paper, the MUSIC detector was filled with methane gas at a pressure of $200 \mathrm{mbar}$. Methane is a fast counting gas with drift velocities up to $10 \mathrm{~cm} / \mu \mathrm{s}$ [18]. The electric field to pressure $(\varepsilon / p)$ ratios in the MUSIC detector were between 0.5 to $1 \mathrm{~V} /(\mathrm{cm}$ mbar). The gas pressure in the ionization chamber was controlled by a pressure-regulated gas handling system. This gas handling system has a high precision pressure gauge and magnetic valves which, together with a vacuum pump, establish a constant flow of the counting gas. In this way, the pressure is maintained at a fixed preset value preventing the detector/target gas from degradation. The pressure gauge of the gas-handling system was calibrated against a high-precision Wallace \& Tiernan gauge [19]. The gas temperature was also monitored in order to obtain the density needed for calculating the cross sections. The energy deposited in the detector by an incident beam-intensity of approximately $10^{4}$ particles/s corresponds to a heat load of less than $1 \mu \mathrm{J}$, which is too low to cause a change in temperature.

The correct identification of fusion events with the MUSIC detector requires that only one incoming particle is inside each "beam bucket". While this is usually not a problem for secondary beams that experience a reduction in intensity by a factor of $10^{5-6}$ relative to the primary beam incident on the production target, for stable beams the intensity needs to be reduced accordingly. In order to avoid pile-up in the IC, which has typical electron drift times of 1-2 $\mu \mathrm{s}$, we used the RF beam-sweeper at ATLAS. This device was operated as a chopper and increased the time between two consecutive beam bunches from 82 ns to $4-10 \mu \mathrm{s}$.

\subsection{Energy measurement}


For obtaining an excitation function, an energy value must be associated to each strip. Normally this is done by using energy-loss tables from the literature (e.g. LISE++ [20], SRIM [21]). It is also known, however, that these tables differ in their predictive power by up to $10 \%$ [21]. In order to measure the correct energy loss, MUSIC was mounted in front of the Enge-Split-Pole spectrograph (SPS) that was calibrated with $\alpha$ particles from a ${ }^{228} \mathrm{Th}$ source. Beams of ${ }^{13,15} \mathrm{C}$ were passing through the MUSIC detector filled with $\mathrm{CH}_{4}$ at various pressures. The experimental energy loss values measured in the spectrograph are shown as the solid bars in Fig. 5, compared to the predictions of the codes LISE++ (v. 9.7) and SRIM (v. 2013). For a ${ }^{13} \mathrm{C}$ beam the LISE++ parameterization shows the best agreement, while for ${ }^{15} \mathrm{C}$ SRIM gives a slightly better overlap with the data. We used the LISE++ values for those cases where no spectrograph calibration has been performed. From these (calculated) energy losses, the energy value corresponding to each strip is obtained taking into account their finite size and assuming that fusion occurs in the middle of the strip.

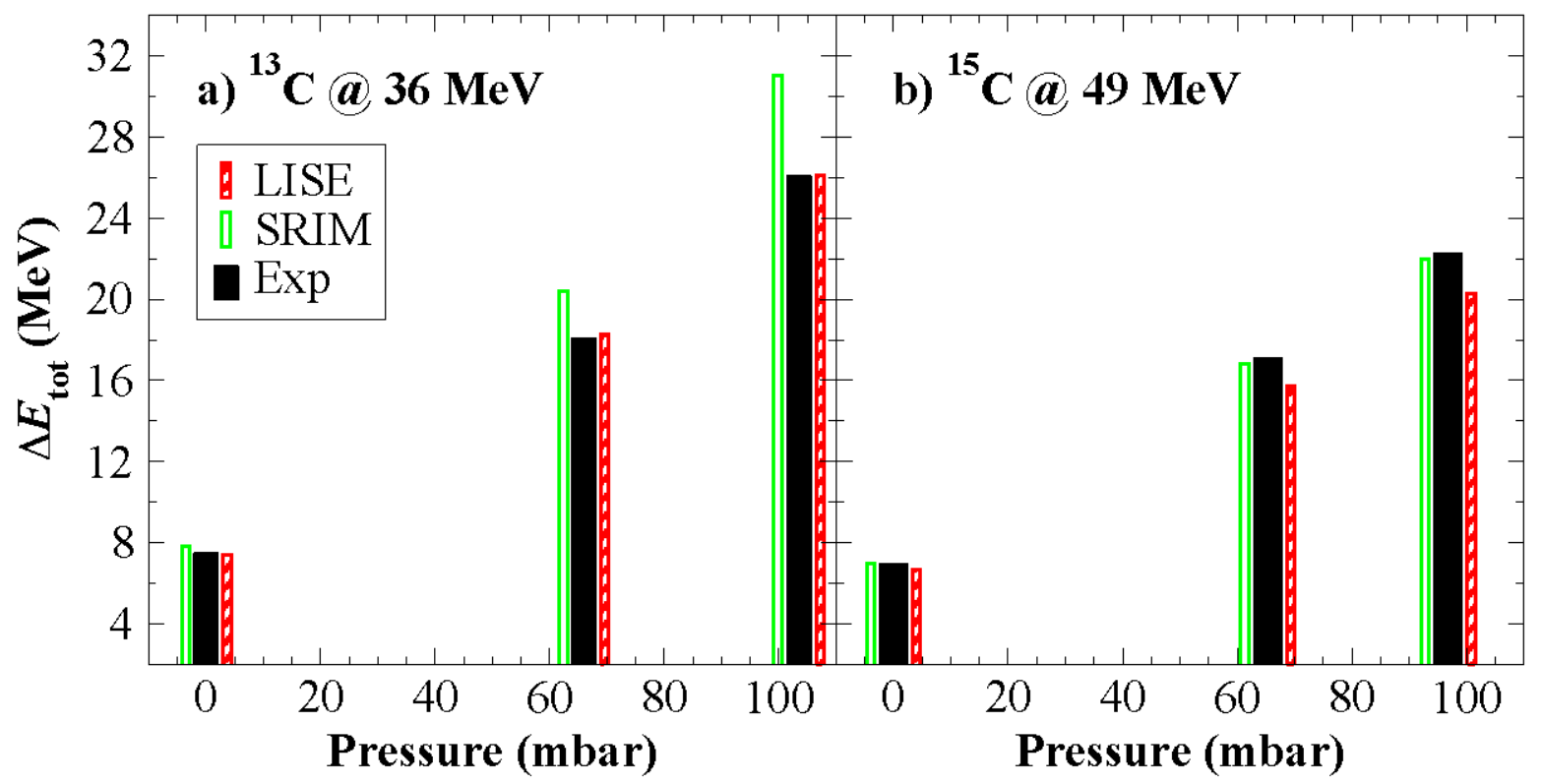

Fig. 5 (color online). Comparison between calculated (LISE++ 9.7, SRIM 2013) and experimental total energy losses in the MUSIC detector, as a function of the gas pressure.

\subsection{Particle identification in experiments with secondary beams}

At the present radioactive beam facilities, the secondary beams have typically contaminants of various sorts. Therefore, it is necessary to tag each incoming particle according to its mass and $Z$ number. At ATLAS, the beam is pulsed with a period of $82 \mathrm{~ns}$. The measurement of the time of arrival of the beam particle in the IC with respect to the RF signal from the accelerator allows us to identify the various particle groups obtained in the production of secondary beams [15].

Examples of beam contaminants of radioactive beams produced via the inverse $(\mathrm{p}, \mathrm{n})$ or $(\mathrm{d}, \mathrm{p})$ reactions can be found in the literature $[15,22]$. In this paper we briefly describe two examples of the particle 
identification procedure. The ${ }^{10} \mathrm{C}$ beam was produced with the in-flight technique via the ${ }^{1} \mathrm{H}\left({ }^{10} \mathrm{~B},{ }^{10} \mathrm{C}\right) \mathrm{n}$ reaction by bombarding a hydrogen-filled gas cell with an $80 \mathrm{MeV}{ }^{10} \mathrm{~B}^{4+}$ beam from the ATLAS accelerator. The main contaminant observed in this case came from the primary ${ }^{10} \mathrm{~B}$ beam which could be suppressed using time-of-flight and energy loss signals in the control strip $\mathrm{S}_{0}$ of the ionization chamber (see Fig. 6a). The other contaminant was ${ }^{15} \mathrm{~N}^{6+}$ from the ECR ion source and was well separated from ${ }^{10} \mathrm{C}$. The ${ }^{15} \mathrm{C}$ beam was produced via the $\mathrm{d}\left({ }^{14} \mathrm{C},{ }^{15} \mathrm{C}\right) \mathrm{p}$ reaction by bombarding a deuterium-filled gas cell with a ${ }^{14} \mathrm{C}$ beam from the tandem accelerator at ATLAS, using a ${ }^{14} \mathrm{C}$ sputter target in the negative ion source. Since nitrogen does not form negative ions a pure ${ }^{14} \mathrm{C}$ beam is injected into the accelerator. The beam contaminants in this case included ${ }^{14} \mathrm{C}$ from the production beam as well as ${ }^{15} \mathrm{~N}$ particles produced via the $\mathrm{d}\left({ }^{14} \mathrm{C},{ }^{15} \mathrm{~N}\right)$ n reaction. Again a measurement of the time of arrival of the beam bunches at MUSIC together with the energy loss in the control strip $S_{0}$ allowed a separation of the particles of interest from the contaminants as shown in Fig. 6 b.
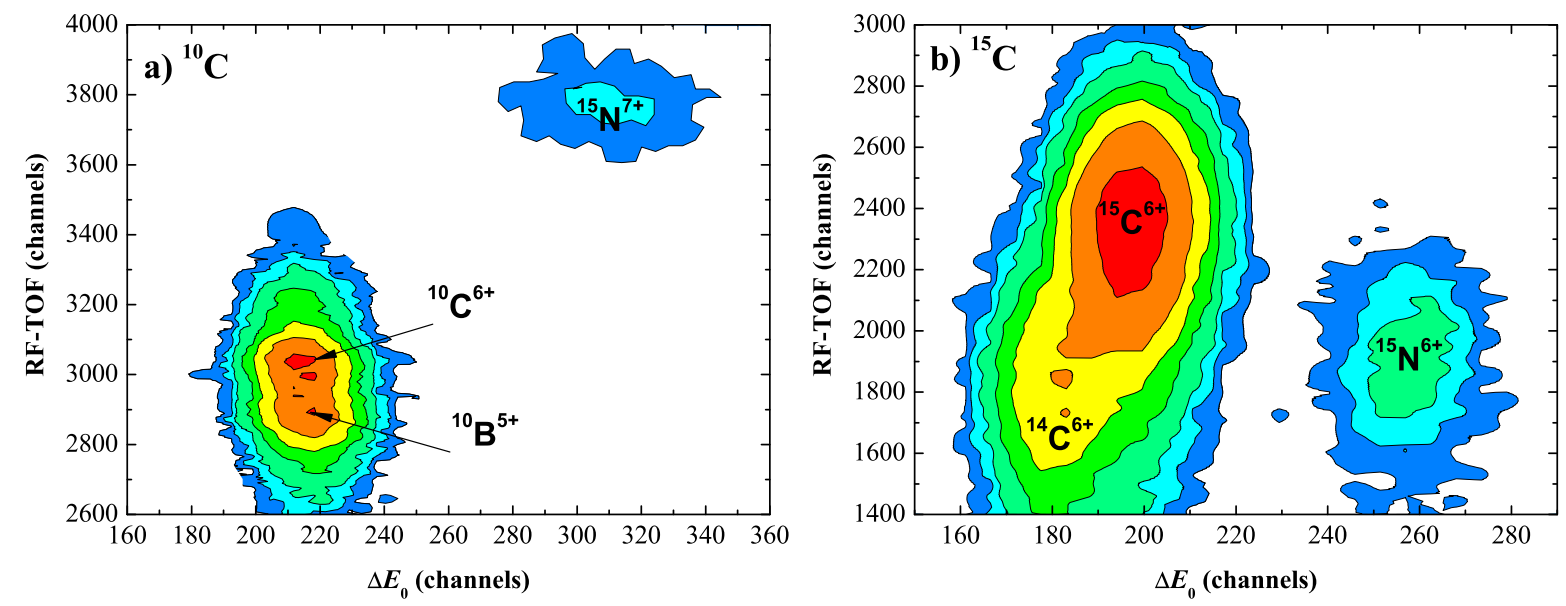

Fig. 6 (color online). $\Delta E_{0}$ vs. RF-ToF spectra showing the separation between the secondary beams and the contaminants generated by the primary beams for a) $43 \mathrm{MeV}{ }^{10} \mathrm{C}$ and b) $49 \mathrm{MeV}{ }^{15} \mathrm{C}$ secondary beams.

\section{Experimental Results}

In order to demonstrate the capabilities of the MUSIC technique we discuss the experimental results of the ${ }^{13} \mathrm{C}+{ }^{12} \mathrm{C}$ and ${ }^{10,15} \mathrm{C}+{ }^{12} \mathrm{C}$ fusion reactions using a stable ${ }^{13} \mathrm{C}$ and secondary ${ }^{10,15} \mathrm{C}$ beams. The main experimental parameters are summarized in Table 1.

The analysis of the signals from the MUSIC detector was performed on an event-by-event basis. The analysis consisted of counting the number of beam-like particles needed for normalization and of the fusion events identified by their large energy loss, their length (typically covering 3-5 strips) and the lack of energy-loss signals in the strips further downstream (see Sect. 2). Due to those requirements, the detector provided only 11-13 data points. The effective target thickness needed for the cross section was derived from the width of the individual anode strips and the pressure and the temperature of the $\mathrm{CH}_{4}$ gas in the detector. 
Table 1. Main parameters of the MUSIC experiments: beam isotope, incident beam energy, ionization gas pressure, and center of mass energy range for the ${ }^{A} \mathrm{C}+{ }^{12} \mathrm{C}$ system measured in strips 1-16.

\begin{tabular}{cccc}
\hline \hline Beam & $\begin{array}{c}\boldsymbol{E}_{\text {lab }} \\
(\mathbf{M e V})\end{array}$ & $\begin{array}{c}\text { Pressure } \\
(\mathbf{m b a r})\end{array}$ & $\begin{array}{c}\boldsymbol{E}_{\text {c.m. }} \text { range } \\
(\mathbf{M e V})\end{array}$ \\
\hline${ }^{13} \mathrm{C}$ & 36 & 200 & $7.6-14.0$ \\
& 45 & 200 & $9.9-18.3$ \\
${ }^{10} \mathrm{C}$ & 43 & 240 & $9.5-19.9$ \\
${ }^{15} \mathrm{C}$ & 49 & 200 & $8.9-18.3$ \\
\hline \hline
\end{tabular}

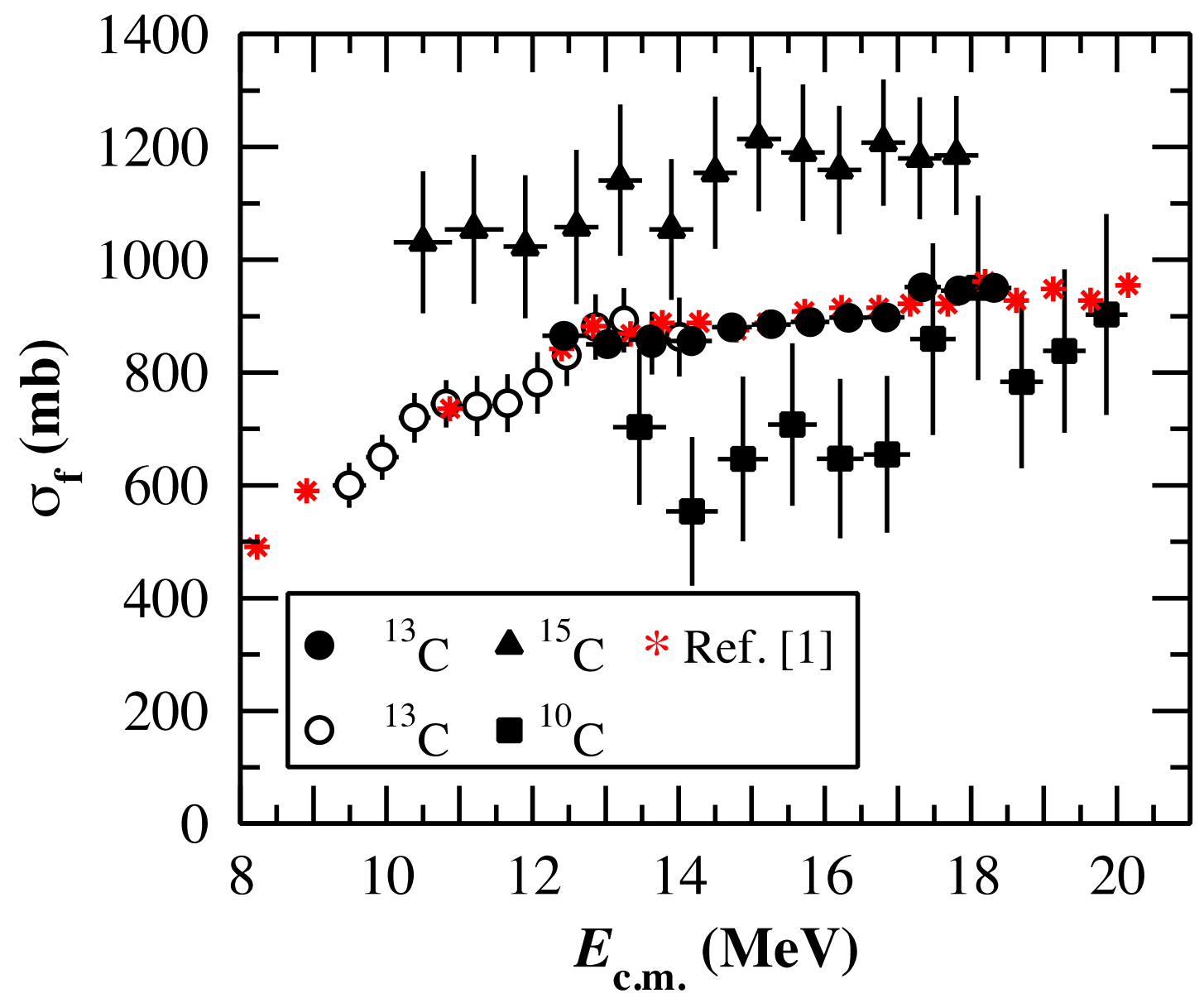

245 Fig. 7 (color online). Experimental ${ }^{10,13,15} \mathrm{C}+{ }^{12} \mathrm{C}$ fusion excitation functions from the present work. For the ${ }^{13} \mathrm{C}+{ }^{12} \mathrm{C}$ 246 case two different incident energies were used (open circles correspond to $36 \mathrm{MeV}$ and filled circles correspond to $247 \quad 45 \mathrm{MeV}$ ). The experimental data from Ref. [1] for the ${ }^{13} \mathrm{C}+{ }^{12} \mathrm{C}$ system are also included for comparison. 
Since precise measurements of these parameters were obtained, the overall uncertainty in the cross sections is determined by the statistics of the experiment.

The uncertainty in the energy values of the excitation function originates from the finite size of the strips since fusion can occur at different positions, and hence with different energies, in the same strip. For this reason, we report the energy uncertainty as the calculated energy loss in each strip. For the current experiments it varied between $2 \%$ and $6 \%$ of the c.m. energy, increasing with strip number.

The fusion cross sections measured for the systems ${ }^{10,13,15} \mathrm{C}+{ }^{12} \mathrm{C}$ are shown in Fig. 7. For the ${ }^{13} \mathrm{C}+{ }^{12} \mathrm{C}$ case two measurements with different incident beam energies (36 and $45 \mathrm{MeV}$ ), but the same gas pressure, were performed (see Table 1). The intention of these first test measurements was to study the operation of the detector and the consistency of the analysis process with a set of overlapping excitation functions for a system that has been measured using one of the standard techniques. The results are compared to the data from Ref. [1] in Fig. 7b and an excellent agreement is observed.

The data corresponding to ${ }^{10,15} \mathrm{C}+{ }^{12} \mathrm{C}$ are the first fusion measurements obtained for these systems. The shapes of the excitation functions are all similar, with a plateau at the higher energies and a fall-off of the cross sections towards lower energies. Because of the limited statistics achieved with radioactive beams, the uncertainties in $\sigma_{\mathrm{f}}$ for ${ }^{10,15} \mathrm{C}+{ }^{12} \mathrm{C}$ are higher than for the stable-beam reaction ${ }^{13} \mathrm{C}+{ }^{12} \mathrm{C}$, reaching about $12 \%$ in the former case.

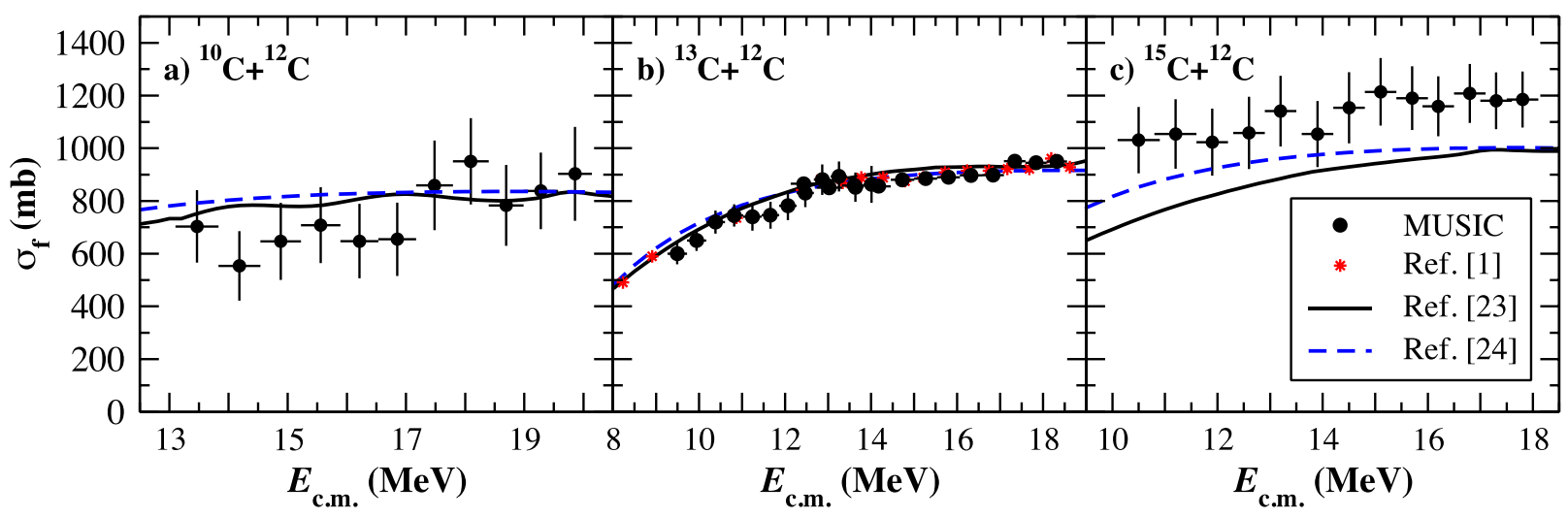

Fig. 8 (color online). Comparison of MUSIC experimental data with theoretical calculations. Solid circles: experimental data obtained with the MUSIC detector. Solid lines: theoretical cross sections from coupled-channels calculations [23]. Dashed lines: predictions from a barrier penetration model using the São Paulo potential [24].

In Fig. 8 we have compared the measured cross sections with various theoretical predictions. The solid lines are the predictions from coupled-channels calculations. These calculations were done using a double-folding potential and including couplings to one- and two-phonon excitations as well as mutual quadrupole and octupole excitations in projectile and target [23]. The dashed lines are the results of a barrier-penetration model based on the São Paulo potential [24] (previously shown as astrophysical Sfactors in Ref. [25]). In the ${ }^{13} \mathrm{C}+{ }^{12} \mathrm{C}$ case (Fig. 8b), the agreement between both models and the experimental data is excellent. On the other hand, in the radioactive beam cases, the São Paulo potential predictions are about 10\% above the coupled-channels results. The two models overestimate the 
experimental data for ${ }^{10} \mathrm{C}+{ }^{12} \mathrm{C}$ in the $E_{\text {c.m. }}=13-17 \mathrm{MeV}$ energy range and show better agreement at higher energies. Both models underestimate the experimental cross sections in the ${ }^{15} \mathrm{C}+{ }^{12} \mathrm{C}$ case.

\section{Summary}

We have developed and tested a new high-efficiency, active-target system in the form of a small multisampling ionization chamber. This detector allowed us to determine a large part of the excitation function in a single measurement, thus saving considerable running time and eliminating difficulties with the relative normalization of the cross sections. Since the detector operates at low beam intensities, it is ideally suited for experiments with the radioactive ion beams that are available at present-day facilities. The separation of beam contaminants in experiments with these beams was achieved by measuring the energy loss and the time-of-flight of the secondary radioactive beam particles.

The performance of the MUSIC detector was tested in experiments with stable ${ }^{13} \mathrm{C}$ and radioactive ${ }^{10,15} \mathrm{C}$ beams. The agreement with data from the literature for the ${ }^{13} \mathrm{C}$ case was excellent. Monte Carlo simulations using the code FLUKA were used to understand how to correctly identify the various nuclear processes and to develop an algorithm to extract the fusion cross sections. In this way, we obtained a full characterization of the detector that we can use to predict its response in future experiments.

The main disadvantage of the MUSIC detector in its present form is the relatively low beam rate it can tolerate in order to avoid pile-up effects that could be interpreted as fusion events. At a pulsed beam accelerator such as ATLAS, this is achieved by having at most one particle in each bunch and separating the bunches with a sweeper system. In this way, the beam bunches arrive to the detector at intervals of about $5 \mu \mathrm{s}$, which is the typical drift time in the ionization chamber. Improving the count rate capability of the MUSIC detector by introducing a "gating" grid [26, 27] is planned in the future. Other upgrades include the addition of parallel-plate-avalanche counters at the entrance and exit for better timing signals and measurements of the vertical drift times.

In these first experiments we have demonstrated that the MUSIC detector is a powerful device especially for the measurement of fusion reactions with radioactive beams. Measurements involving other isotopically pure gases ( $\mathrm{He}, \mathrm{Ne}, \mathrm{Ar})$ have already started.

\section{Acknowledgments}

We thank the operations crew of the ATLAS accelerator for providing the beams used in these experiments. This work was supported by the US Department of Energy, Office of Nuclear Physics under contract No. DE-AC02-06CH11357, and the Consejo Nacional de Investigaciones Científicas y Técnicas (CONICET), Argentina.

\section{References}


312 [1] D. G. Kovar et al., Phys. Rev. C 20, 1305 (1979).

313 [2] X. D. Tang et al., J. Phys.: Conf. Ser. 381, 012120 (2012).

314 [3] B. Dasmahapatra et al., Nucl. Phys. A 565, 657-670 (1993).

315 [4] R. A. Dayras et al., Nucl. Phys. A 265, 153 (1975).

316 [5] T. Spillane et al., Phys. Rev. Lett. 98, 122501 (2007).

317 [6] M. Notani et al., Nucl. Phys. A 834, 192 (2010).

318 [7] D. Di Gregorio et al., Phys. Lett. B 176, 322 (1986).

319 [8] J. O. Fernández Niello et al., Phys. Rev. C 43, 2303 (1991).

320 [9] A. J. Pacheco et al., Comp. Phys. Comm. 52, 93-102 (1988).

321 [10] M. Alcorta et al., Phys. Rev. Lett. 106, 172701 (2011).

322 [11] K. E. Rehm et al., Nucl. Inst. Meth. A 344, 614-622 (1994), and references therein.

323 [12] W. B. Christie et al., Nucl. Inst. Meth. A 255, 466-476 (1987).

324 [13] K. Kimura et al., Nucl. Inst. Meth. A 297, 190-198 (1990).

325 [14] Y. Mizoi et al., Nucl. Inst. Meth. A 431, 112-122 (1999).

326 [15] B. Harss et al., Rev. Sci. Instr. 71, 380-387 (2000).

327 [16] Mesytec GmbH \& Co., http://www.mesytec.com/nuclear.htm.

328 [17] G. Battistoni et al., AIP Conf. Proc. 896, 31-49 (2007).

329 [18] Th. Berghoefer et al., Nucl. Inst. Meth. A 525, 544-552 (2004).

330 [19] FLW Inc., http://www.wallaceandtiernan-usa.com/absolute-pressure-gauges-1500-8A.html.

331 [20] O. B. Tarasov and D. Bazin, Nucl. Instr. Meth. B 266, 4657-4664 (2008). http://lise.nscl.msu.edu.

332 [21] J. F. Ziegler et al., Nucl. Inst. Meth. B 268, 1818-1823 (2010). http://www.srim.org.

333 [22] X. D. Tang et al., Phys. Rev. C 81, 045809 (2010).

334 [23] H. Esbensen et al., Phys. Rev. C 84, 064613 (2011), and private communication.

335 [24] D. G. Yakovlev et al., Phys. Rev. C 82, 044609 (2010).

336 [25] P. F. F. Carnelli et al., Phys. Rev. Lett. 112, 192701 (2014).

337 [26] P. Nemethy et al., Nucl. Inst. Meth. A 212, 273-280 (1983). 
338 [27] T. Hashimoto et al., Nucl. Inst. Meth. A 556, 339-349 (2006). 